\title{
WHEN ARE PROPER SUBGROUPS OF LCA GROUPS CONTAINED IN MAXIMAL ONES?
}

\author{
M. A. KHAN
}

\begin{abstract}
ABSTRACr. In this note we determine (1) the class of LCA groups for which every proper closed subgroup is contained in a maximal subgroup, (2) the class of LCA groups for which every proper dense subgroup is contained in a maximal subgroup, and (3) the class for which every proper subgroup is contained in a maximal one. We also determine when both an LCA group and its dual have these properties.
\end{abstract}

1. Introduction. Let $G$ be an LCA group. We shall say that $G$ possesses property C if every proper closed subgroup of $G$ is contained in a maximal subgroup. $G$ possesses property $\mathrm{D}$ if every proper dense subgroup is contained in a maximal subgroup, and $G$ has property $\mathrm{E}$ if every proper subgroup is contained in a maximal one. $B(G)$ denotes the subgroup of compact elements; $D(G)$, a minimal divisible extension of $G$, topologized in the usual manner so that $G$ is an open subgroup. $G_{0}$ is the component of the neutral element, and $\hat{G}$, the character group

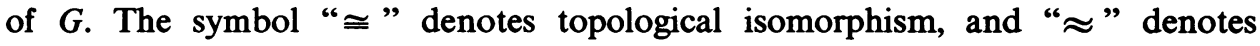
isomorphism in the algebraic sense.

For an abelian group $A$ (topological or not), $T(A)$ and $U(A)$ denote respectively the maximal torsion subgroup and the first Ulm subgroup of $A$ (see [3]). $r(A)$ is the rank of $A$. A subgroup of $A$ is a maximal subgroup iff it is of prime index. We define an abelian group $A$ to be totally reduced if every nontrivial homomorphic image of $A$ is reduced. As we show below, totally reduced abelian groups provide just the right kind of group-theoretic machinery to tackle the problems of this paper.

We shall use the quasicyclic groups $Z\left(p^{\infty}\right)$ and the group of additive rationals $Q$. The well-known LCA groups which we shall mention are the circle group $T$, the additive real numbers $R$, the additive group of $p$-adic integers $J_{p}$ (all with their usual topologies), and the discrete group of integers $Z$. For other notation and terminology, we follow [3] and [4].

Finally, we would like to point out that the material in this paper is related to some structural and measure-theoretic properties of LCA groups discussed in [2], [5], [6] and [7], and that partial results concerning the questions raised herein have appeared in [6, Theorem 1.10 and Corollary 1.11].

Received by the editors November 19, 1980.

1980 Mathematics Subject Classification. Primary 22B05; Secondary 20K27, 20K99.

Key words and phrases. Maximal subgroups, reduced, totally reduced, divisible, abelian groups, locally compact abelian, $p$-adic integers, quasicyclic. 
2. Results. Our first object is to determine the structure of totally reduced abelian groups. We start with a result that brings out the relevance of this class of groups to the theme of this paper.

Proposition 1. Every proper subgroup of an abelian group is contained in a maximal subgroup iff it is totally reduced.

Proof. Let $A$ be totally reduced and let $H$ be a proper subgroup. Since $A / H$ is reduced, it contains a maximal subgroup [3, Exercise 1, p. 99]. Hence, $H$ is contained in a maximal subgroup of $A$. Conversely, let $L$ be a proper subgroup of $A$ such that $A / L$ is not reduced. Then $L$ is contained in a proper subgroup $M$ such that $A / M$ is divisible, and this subgroup $M$ is not contained in any maximal subgroup. Hence, $A$ must be totally reduced, and the proof is complete.

Having done this, we can now move towards our first goal with a clearer conscience.

PROPOSITION 2. Subgroups and quotient groups of totally reduced abelian groups are totally reduced.

Proof. Let $\boldsymbol{A}$ be totally reduced and let $\boldsymbol{H}$ be a proper subgroup of $\boldsymbol{A}$. If $\boldsymbol{H}$ is not totally reduced, $H$ contains a proper subgroup $K$ such that $H / K$ is divisible. Since $A / H \approx(A / K) /(H / K)$, it follows that $A / K$ is not reduced, contradicting the hypothesis. Hence, $H$ is totally reduced. The assertion about quotient groups follows from the definition of totally reduced groups.

Corollary 1. An abelian group $A$ is totally reduced iff both $T(A)$ and $A / T(A)$ are totally reduced.

Proof. The "necessity" part follows from Proposition 2. For the "sufficiency", suppose $T(A)$ and $A / T(A)$ are totally reduced, and $H$ is an arbitrary proper subgroup of $A$. If $H+T(A)=A$, then $A / H \approx T(A) / H \cap T(A)$, and so is reduced. If $H+T(A) \neq A$, then $A /(H+T(A))$ is a homomorphic image of $A / T(A)$, and so must be reduced. This implies that $H+T(A)$ is contained in a maximal subgroup of $A$. Clearly the same is true for $H$, and the proof is complete.

In view of Corollary 1 , it suffices to determine the structure of those totally reduced groups which are either torsion or torsion-free.

Proposition 3. Let $A$ be an abelian group.

(a) If $A$ is a torsion group, then $A$ is totally reduced iff each p-component of $A$ is bounded.

(b) If $A$ is torsion-free, then $A$ is totally reduced iff $r(A)$ is finite, and for every free subgroup $M$ of $A$ such that $r(A)=r(M)$, each $p$-component of $A / M$ is finite.

Proof. (a) Suppose a $p$-component $A_{p}$ of $A$ is not bounded. Then $A_{p}$ contains a proper basic subgroup, say $H$, and $A_{p} / H$ is divisible [3, Corollary 35.4]. But this implies that $A$ is not totally reduced. Conversely, if $A_{p}$ is bounded for every prime $p$, then no homomorphic image of $A$ is $\approx Z\left(p^{\infty}\right)$ for any prime $p$. Hence, in this case, $A$ is totally reduced. 
(b) First let $A$ be totally reduced. Suppose, if possible, that $r(A)$ is infinite and let $N$ be a free subgroup generated by a countably infinite independent subset of $A$. By an obvious construction, we can get a subgroup $S$ of $N$ such that $N / S \approx$ $\bigoplus_{k=1}^{\infty} Z\left(p^{k}\right)$. As pointed out in the proof of (a), this group has a proper basic subgroup and so Proposition 2 implies that $A$ is not totally reduced. Hence, $r(A)$ must be finite. Now, let $M$ be a free subgroup with $r(M)=r(A)$. Then for every prime $p$, the $p$-component of $A / M$ is a reduced subgroup of a direct sum of finitely many copies of $Z\left(p^{\infty}\right)$. Hence, it is finite. Conversely, suppose $A$ has a nonzero divisible homomorphic image. Then $A$ has a nonzero torsion divisible homomorphic image, $A / H$ say, where $H$ contains a free subgroup, say $M$, with $r(A)=$ $r(M)$. But this contradicts the hypothesis about the $p$-components of $A / M$. Hence, $A$ is totally reduced and the proof is complete.

Using duality, we deduce a result of some interest about compact abelian groups.

Proposition 4. A compact abelian group $G$ contains no nontrivial closed torsionfree subgroup iff $\hat{G}$ is totally reduced.

Proof. The discrete group $\hat{G}$ is totally reduced iff it has no homomorphic image $\approx Z\left(p^{\infty}\right)$ for any prime $p$. By $[4,25.8]$, a compact abelian group contains no nontrivial closed torsion-free subgroup iff it contains no copy of $J_{p}$ for any prime $p$. The result follows by duality.

We are now in business. It is clear that an LCA group has property $\mathrm{E}$ iff it has properties $C$ and D both. We could deal with properties $C$ and $D$ first, and use them to study property $\mathrm{E}$. However, a direct treatment of property $\mathrm{E}$ via totally reduced groups is simpler and more natural. First, we make a remark whose truth is obvious, and which we shall use in the sequel.

REMARK 1. Let $G$ be an LCA group. Then $T(G)$ is open iff $G_{0}$ is trivial and $T(G)=B(G)$.

We are now ready for our first theorem.

THEOREM 1. An LCA group $G$ possesses property $\mathrm{E}$ iff $T(G)$ is open and $\simeq M \times$ $N$, where $M$ is a bounded LCA group, $N$ is a discrete, torsion, totally reduced group, and $G / T(G)$ is a (discrete torsion-free) totally reduced group.

Proof. By Proposition 1, it follows that an LCA group has property E iff it is totally reduced. Now, $G$ is clearly totally reduced in case the structure of $G$ is as given in the hypothesis. Conversely, let $G$ be totally reduced. Then $G$ is totally disconnected. If $T(G)$ is a proper subgroup of $B(G)$, then $B(G)$ contains a compact monothetic (necessarily uncountable) subgroup $H \cong$ a topological direct product $\Pi_{p} H_{p}$, where $H_{p}$ is either $J_{p}$ or a cyclic $p$-group or trivial $[4,25.16]$. Since $H$ is uncountable and $T(H)$ is countable, $H$ is not totally reduced. Hence, $T(G)=$ $B(G)$, so that $T(G)$ is open by Remark 1. Also $G / T(G)$ is totally reduced. Let $\bigoplus G_{p}$ be the decomposition of $T(G)$ into $p$-components as an abstract group. Let $K$ be a compact, open, $n$-bounded subgroup. Let $M$ be the (open) subgroup generated by those $p$-components of $T(G)$ for which $p$ divides $n$, and let $N$ be the discrete 
complement of $M$. Since $M$ is open, we conclude that $T(G) \cong M \times N$, and the proof is complete.

As remarked in the proof, Theorem 1 describes the structure of totally reduced LCA groups.

The following two corollaries follow directly from Theorem 1.

COROLlaRY 2. A torsion-free LCA has property $\mathrm{E}$ iff it is discrete and totally reduced.

COROLlaRY 3. A compact abelian group has property E iff it is torsion (hence, bounded).

We turn next to property $D$.

TheOReM 2. An LCA group $G$ has property $\mathrm{D}$ iff $T(G)$ is open.

Proof. Suppose $T(G)$ is open and let $H$ be a proper dense subgroup of $G$. $T(G)$ contains a compact, open, bounded subgroup $K$, say $m K=\{0\}$. Then $H$ contains $m G$ [5, Lemma 5.5]. Since $G / m G$ is totally reduced, it is obvious that $H$ is contained in a maximal subgroup of $G$.

Conversely, suppose $G$ has property $D$. Then $T$ cannot be a homomorphic image of $G$, for this would contradict property D. Hence, $G$ is totally disconnected. In view of Remark 1 , it is enough to show that $T(G)=B(G)$. Suppose not, then $B(G)$ contains an infinite cyclic subgroup. Hence $D(G)$ contains a copy of $Q$ with the nondiscrete topology. By [1, Theorem 2], $\bar{Q}$ is divisible. Hence, we have $D(G)=\bar{Q}$ $+L$, for some subgroup $L$. Now $(Q+L) \cap G$ is clearly a proper dense subgroup of $G$, and

$$
G /((Q+L) \cap G) \approx(G+Q+L) /(Q+L)=D(G) /(Q+L),
$$

which is divisible. This contradicts property D. Hence, $T(G)=B(G)$ and the proof is complete.

COROllaRY 4. If an LCA group $G$ has property $\mathrm{D}$, and $H$ is a closed subgroup, then both $H$ and $G / H$ have property $D$.

Proof. For $H$, we note that $T(H)=H \cap T(G)$. For $G / H$, we observe that the image of the open subgroup $H+T(G)$ under the natural homomorphism onto $G / H$ is contained in $T(G / H)$. Theorem 2 completes the proof.

Corollary 5. A compact abelian group has property D iff it is bounded.

Proof. If $G$ is compact, $T(G)$ is open iff $G=T(G)$.

COROLlARY 6. Every torsion LCA group has property D.

Proof. Obvious.

Remark 2. Theorem 2 needs some explanation. Suppose $G$ is a torsion, divisible LCA group. Then $G$ is as far from being totally reduced as it can be; it just contains no maximal subgroups. How then do the proper dense subgroups of $G$ manage to be contained in maximal subgroups? Well, just by not existing. In fact, a 
nondiscrete LCA group $G$ contains no proper dense subgroups iff $T(G)$ is open and $p G$ is open for every prime $p$ [5, Corollary 5.1].

Before passing on to our next theorem, we insert a proposition, which will simplify our proofs.

Proposition 5. An LCA group contains no nontrivial closed torsion-free subgroup iff it contains no copy of $Z$ or $J_{p}$.

Proof. A torsion-free LCA group is of the form $R^{n} \times M \times N$, where $M$ is a topological direct product of copies of $\left(Q_{d}\right)^{\wedge}$ (where $Q_{d}$ is $Q$ taken discrete), and $N$ is a totally disconnected torsion-free group [4, 25.30(c) and 25.8]. The proof is now obvious.

THEOREM 3. The following are equivalent for an LCA group $G$ :

(a) $G$ has property $\mathrm{C}$.

(b) $G$ is totally disconnected, and $G / H$ is totally reduced for every open subgroup $H$ of $G$.

(c) $\hat{G}$ contains no nonzero closed torsion-free subgroup.

(d) $G$ is totally disconnected and contains a compact, open subgroup $K$ such that $G / K$ is totally reduced.

Proof. (a) implies (b): First, $G_{0}$ must be trivial, for otherwise $T$ would be a homomorphic image of $G$, contradicting (a). The assertion about open subgroups is obvious.

(b) implies (c): Since every element of $\hat{G}$ is compact, it is enough to show that $\hat{G}$ contains no copy of $J_{p}$ [Proposition 5]. But this is clear because $G$ has no discrete homomorphic image $\approx Z\left(p^{\infty}\right)$.

(c) implies (d): Since $\hat{G}$ contains no copy of $Z, G$ is totally disconnected. Let $L$ be a compact, open subgroup of $\hat{G}$ and let $K$ be the annihilator of $L$ in $G$. Then $K$ is a compact, open subgroup such that $G / K$ is totally reduced [Proposition 4].

(d) implies (a): Let $H$ be a proper closed subgroup of $G$. If $H+K$ is a proper subgroup of $G$, then by the condition on $K, H+K$ is contained in a maximal subgroup, and so is $H$. If $H+K=G$, then $G / H \cong K /(H \cap K)$ by [4, 5.33]. Now $K /(K \cap H)$ is compact and totally disconnected, so cannot be divisible. This implies that $H$ is contained in a maximal subgroup, and the proof is complete.

Corollary 7. A torsion LCA group has property $\mathrm{C}$ iff it has property $\mathrm{E}$.

Proof. Since every torsion LCA group has property D [Corollary 6], the assertion is obvious.

Corollary 8. A compact abelian group has property $\mathrm{C}$ iff it is totally disconnected.

Proof. By (c) of Theorem 3.

$J_{p}$ has property $\mathrm{C}$, but is not totally reduced. However, we show below that if $G$ has property $\mathrm{C}$, then $\cap_{n=1}^{\infty} \overline{n G}$ is trivial. In particular, $G$ is reduced. First, we prove a lemma.

LEMMA 1. If $A$ is a totally reduced abelian group, then $U(A)$ is trivial. 
Proof. Suppose not. If there is a $U(A)$-high subgroup $H$, then $A / H$ would be divisible [3, Exercise 12, p. 116]. Hence, $U(A)$ is an essential subgroup. By Proposition 3 and [3, Remark (c), p. 98], we conclude that $A$ must be torsion-free. But then $U(A)$ is divisible [3, Exercise 2, p. 101]. Hence, $U(A)$ must be trivial.

COROLlaRY 9. If an LCA group $G$ has property $\mathrm{C}$, then $\cap_{n=1}^{\infty} \overline{n G}$ is trivial.

Proof. By [4, 24.24], it suffices to show that $\hat{G}$ contains a dense torsion subgroup. Since $\hat{G}=B(\hat{G})$, it is enough to show that the (compact) closure of every infinite cyclic subgroup of $\hat{G}$ contains a dense torsion subgroup. Let $H$ be such a subgroup. Since $\hat{H}$ is discrete and totally reduced, we conclude by Lemma 1 that $H$ does contain a dense torsion subgroup. This completes the proof.

COROLlARY 10. If an LCA group $G$ has property $\mathrm{C}$, and $H$ is a closed subgroup, then both $H$ and $G / H$ have property $\mathrm{C}$.

Proof. By Theorem 3(c), it is clear that $G / H$ has property $C$. Next, suppose $K$ is a proper closed subgroup of $H$ such that $H / K$ is divisible. Then $G / K$ contains a nonzero divisible subgroup, contradicting Corollary 9. Hence, $H$ must have property $\mathrm{C}$.

In the remainder of this note, we investigate when both $G$ and $\hat{G}$ possess one or more of these properties.

THEOREM 4. Let $G$ be an LCA group. Then both $G$ and $\hat{G}$ have property $C$ iff $G_{0}$ is trivial, $G=B(G)$, and $G$ has a compact open subgroup $H$ such that both $G / H$ and $\hat{H}$ are totally reduced.

Proof. Suppose both $G$ and $\hat{G}$ satisfy the property, then $G$ and $\hat{G}$ are totally disconnected [Theorem 3], so $G=B(G)$. Also $G$ has a compact open subgroup $H$ such that $G / H$ is totally reduced [Theorem 3(d)]. Now, $A(\hat{G}, H)$ is an open subgroup of $\hat{G}$, so $\hat{G} / A(\hat{G}, H)$ is totally reduced [Theorem 3(b)]. But this implies $\hat{H}$ is totally reduced. Conversely, the hypothesis implies that both $G$ and $\hat{G}$ are totally disconnected, and that condition (d) of Theorem 3 is satisfied by both $G$ and $\hat{G}$. This completes the proof.

Before passing on to the last theorem of this note, we make an obvious but useful remark.

Remark 3. Let $G$ be an LCA group. Then both $G$ and $\hat{G}$ are torsion groups iff $G$ is bounded. Moreover, in this case $G$ is $n$-bounded iff $\hat{G}$ is $n$-bounded.

THEOREM 5. The following are equivalent for an LCA group $G$.

(a) Both $G$ and $\hat{G}$ have property D.

(b) Both $G$ and $\hat{G}$ have property $\mathrm{E}$.

(c) $G$ is bounded.

Proof. Using Theorems 1 and 2 and Remark 3, the proof follows immediately. 


\section{REFERENCES}

1. D. L. Armacost and W. L. Armacost, On Q-dense and densely divisible LCA groups, Proc. Amer. Math. Soc. 36 (1972), 301-305.

2. W. E. Dietrich, Jr., Dense decompositions of locally compact groups, Colloq. Math. 24 (1972), 147-151.

3. L. Fuchs, Infinite abelian groups. Vol. 1, Academic Press, New York, 1970.

4. E. Hewitt and K. A. Ross, Abstract harmonic analysis. Vol. 1, Die Grundlehren der math. Wissenschaften, Band 115, Academic Press, New York; Springer-Verlag, Berlin, 1963.

5. M. A. Khan, Chain conditions on subgroups of LCA groups, Pacific J. Math. 86 (1980), 517-534.

6. H. L. Peterson, Discontinuous characters and subgroups of finite index, Pacific J. Math. 44 (1973), 683-691.

7. M. Rajagopalan and H. Subrahmanian, Dense subgroups of locally compact groups, Colloq. Math. 35 (1976), 289-292.

Department of Mathematics, KuWaIt University, KuWatt 